\title{
EDDY CURRENT SYSTEM FOR DETECTION OF CRACKING BENEATH BRAIDING IN CORRUGATED METAL HOSE
}

\author{
Buzz Wincheski ${ }^{1}$, John Simpson ${ }^{2}$ and George Hall ${ }^{3}$ \\ ${ }^{1}$ NASA Langley Research Center, Hampton, VA 23681 \\ ${ }^{2}$ Lockheed Martin Space Operations, Hampton, VA 23681 \\ ${ }^{3}$ George Washington University, Washington, D.C. 20052
}

\begin{abstract}
In this paper an eddy current system for the detection of partially-through-the-thickness cracks in corrugated metal hose is presented. Design criteria based upon the geometry and conductivity of the part are developed and applied to the fabrication of a prototype inspection system. Experimental data are used to highlight the capabilities of the system and an image processing technique is presented to improve flaw detection capabilities. A case study for detection of cracking damage in a space shuttle radiator retract flex hoses is also presented.
\end{abstract}

Keywords: Eddy Current, Fatigue Crack, Corrugated Metal Hose, Flex Hose, Space Shuttle PACS: $81.70 . \mathrm{Ex}, 81.40 . \mathrm{Np}, 81.05 . \mathrm{Bx}$

\section{INTRODUCTION}

Corrugated metal hose, also known as flex hose, poses several challenges for quantitative nondestructive evaluation techniques. The complicated structure of the part is shown in Figure 1. In this image the metallic braid has been pulled back to reveal the convoluted thin wall structure of the hose. Partially-through-the-thickness cracking in the corrugated metal hose can easily be masked by the irregular geometry of the part. Inspection of the hose through the braid, as required for any in situ application, further complicates the inspection by adding an irregular metallic layer between the inspector and the critical surface. Coupling of thermal or ultrasonic energy into the part and analysis of the energy flow in the system are daunting tasks, and the large percentage of the surface area covered by the metallic braid limits the effectiveness of even a detailed visual inspection. Leak testing can be effective for through-wall cracking, but no good options have been available for detection of partially-through-the-thickness cracks in a pressurized system. In this work an eddy current technique for the detection of such damage is proposed. The probe design and inspection technique, along with experimental data for the detection of cracking in a typical flex hose component, are presented. 


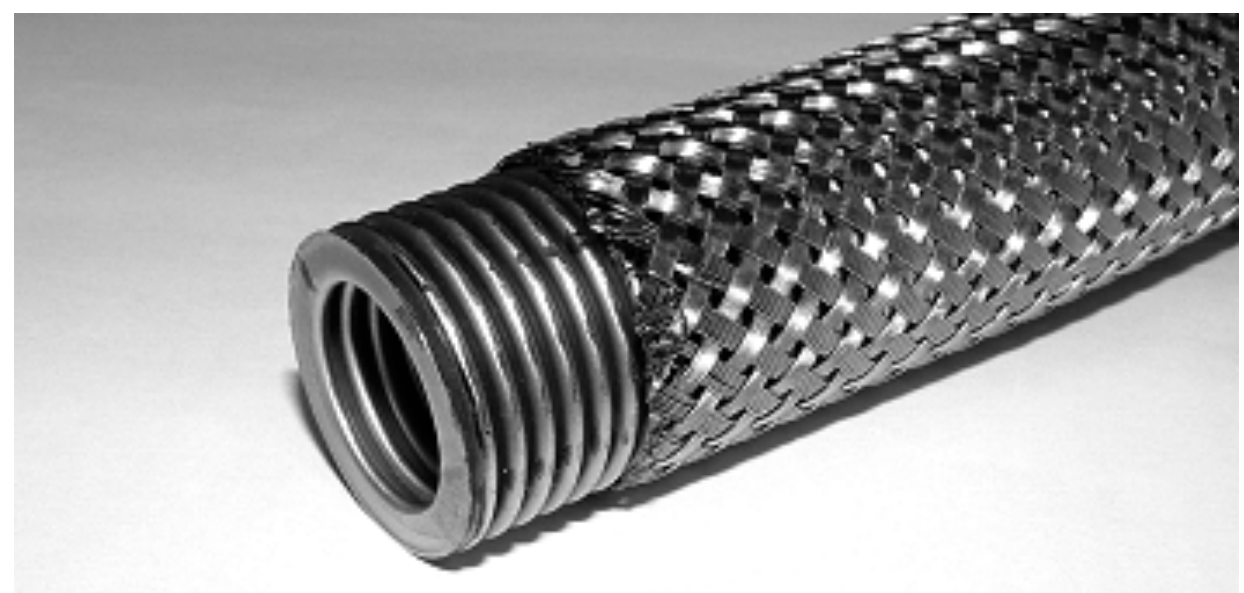

FIGURE 1. Typical flex hose design showing convoluted thin metal wall hose and braided metallic overwrap.

\section{EDDY CURRENT INSPECTION OF CORRUGATED METAL HOSE}

Eddy current technology provides several significant attributes important in overcoming the inspection challenges identified above. Eddy current techniques are well recognized for crack detection capabilities in metallic structures, including thin walled tubing. In addition, multiple systems for detection of flaws in lower layers of metallic multi-layer structures have been developed [1]. Finally, eddy current probe orientation can be used to couple selectively into or away from a feature of the part under test [2].

The initial experimentation on the development of an eddy current technique for the detection of partially-through-the-thickness cracking in flex hose consisted of designing a probe with the correct operating frequency and size to induce a sufficient current density into the hose convolutes. The appropriate operating frequency based upon the required inspection depth and material conductivity can be estimated from the skin depth equation,

$$
B_{x} / B_{0}=e^{-x / \delta}, \quad \delta=1 / \sqrt{\pi f \mu \sigma},
$$

where $B_{x}$ is the field strength at distance $x$ beneath the surface, $\delta=$ the standard depth of penetration, $f=$ excitation frequency, $\mu=$ permeability, and $\sigma=$ electrical conductivity [3]. For a skin depth between 0.4 and $0.8 \mathrm{~mm}$ (distance through the braid and to back surface of hose convolute) in a stainless steel 300 series material, equation (1) estimates a required operating frequency of approximately $0.3-1.0 \mathrm{MHz}$. For probe sizing, the coil diameter was targeted to match the convolute pitch such that the spatial resolution of the probe would correlate with the convolute spacing.

Optimization of the probe orientation was studied to minimize braid effects and maximize coupling to the underlying hose. As can be seen in Figure 2, high pressure flex hose is typically braided with a linear array of stainless steel wires. The resulting braid structure is highly anisotropic in conductivity. Orienting the eddy current probe such that the induced electromotive force (EMF) is always non-parallel to the high conductivity directions of the braid minimizes the shielding effects of the braid. To maximize coupling into the underlying hose, the induced EMF should also be in the plane of the hose. Targeting the detection of circumferential cracks adds a final criterion in that induced currents should be orthogonal to the crack direction for maximum eddy current response from the flaws. Optimization of the probe orientation based upon these criteria led to the 


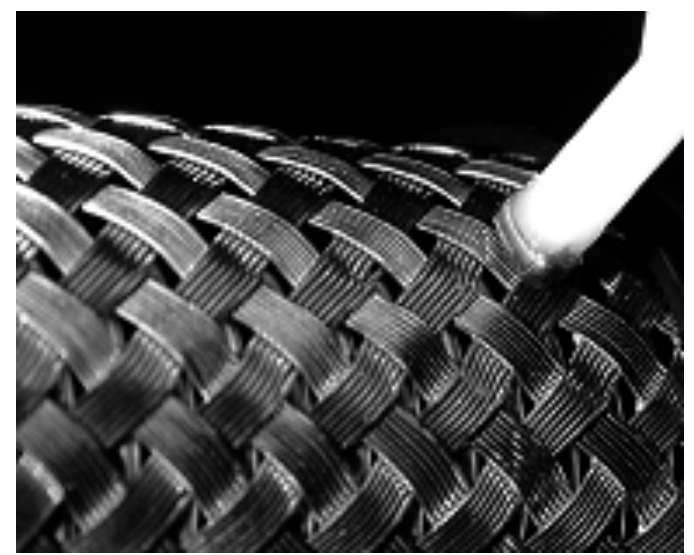

FIGURE 2. Probe orientation implemented to inspect for circumferential cracking in the flex hose convolute.

inspection geometry shown in Figure 2, with the coil axis tangent to the inspection surface and along the convolute direction.

\section{APPLICATION TO SPACE SHUTTLE RADIATOR RETRACT FLEX HOSE}

The effectiveness of the eddy current inspection methodology outlined above was investigated based upon a target inspection of the Space Shuttle Radiator Retract Flex Hose. This component is designed to carry freon to radiators attached to the payload bay doors. The hose is stowed away during take-off and landing but must flex as the payload bay doors open and the radiators are deployed [4-6]. The component is critical for cooling of the orbiter during orbit and has been a source of concern for the shuttle program on multiple occasions [5-6].

A test specimen was fabricated from a spare section of radiator retract flex hose. After removing the flex hose braid to expose the hose convolutes, three laser cut notches were placed in the hose at the crown of the convolute. The notches were each 0.005 " wide x 0.1 " long. The first notch was cut completely through the thickness of the hose while the other two notches were cut to a depth approximately half way through the wall. Figure 3 shows a picture of the hose convolutes with close up views of the three notches.

Initial testing of the eddy current technique was performed by holding the eddy current probe stationary and scanning the hose section. A small air gap of approximately 0.010 " was set between the eddy current coil and the flex hose, with the coil orientation explained above and pictured in Figure 2. The sample was initially scanned with the

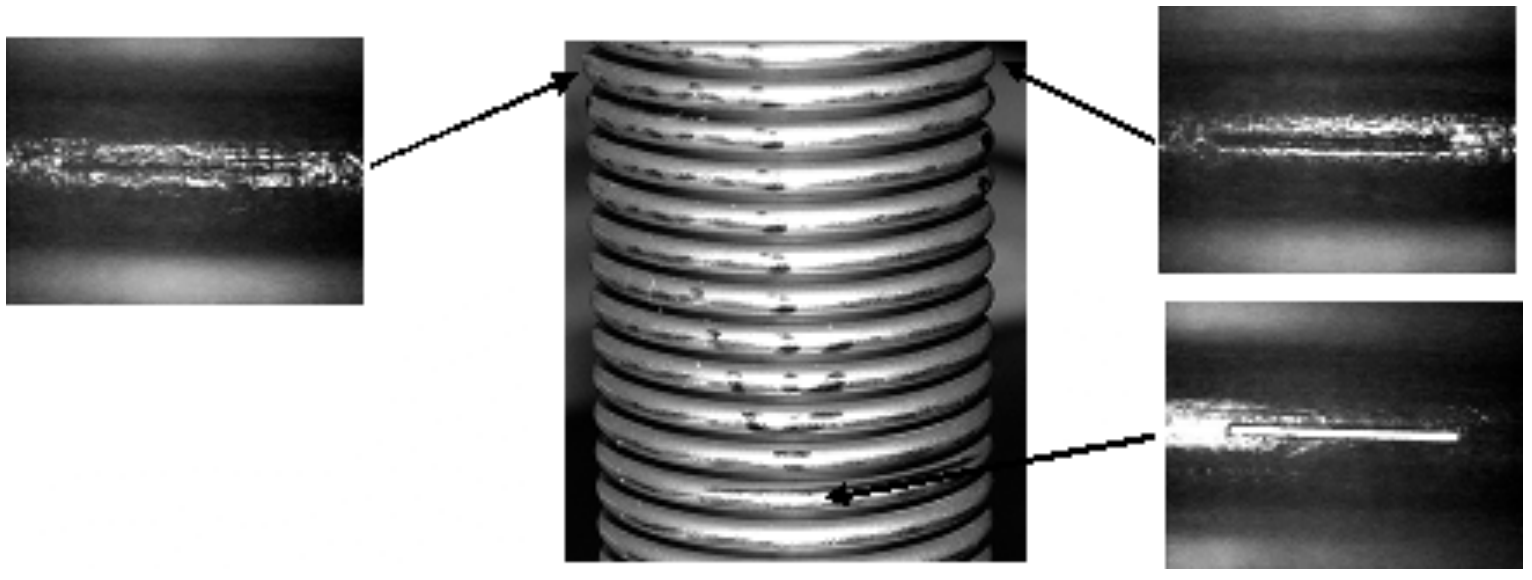

FIGURE 3. Radiator retract verification sample with three laser cut notches, braid removed. 

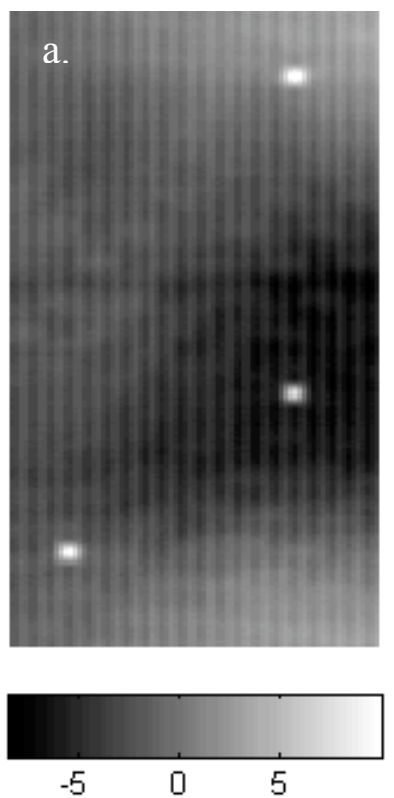
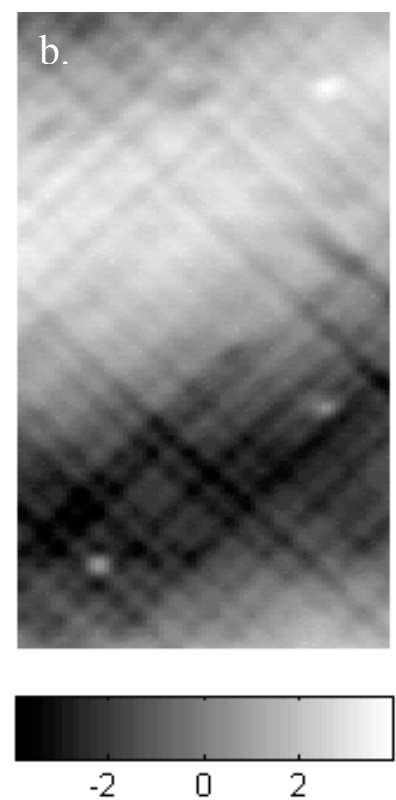
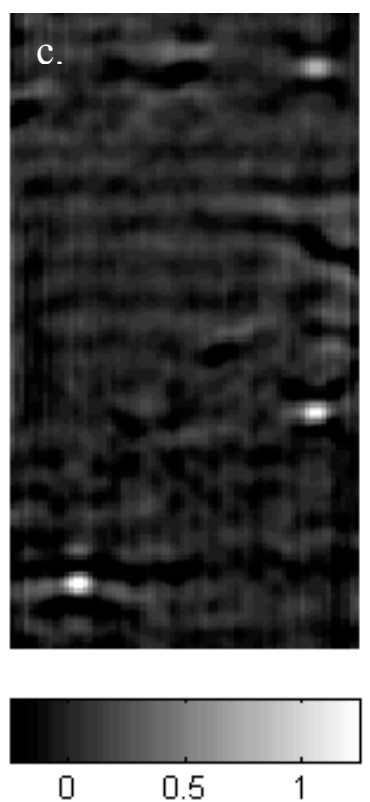

FIGURE 4. Vertical component eddy current C-scan data for (a) validation sample with braid removed, (b) validation sample with braided overwrap in place, and (c) two-dimensional Fourier filter results of (b).

overwrap removed. After optimizing the probe response in this configuration the braided metallic overwrap was placed over the hose and the sample rescanned. In both cases a full 360 degree scan over a 2" length along the hose axis was performed. Figure 4 displays the vertical component eddy current $\mathrm{C}$-scans of the raw data, along with two-dimensional Fourier filter processed results for the scan of the braid overwrapped hose. In the figures the circumferential direction is vertical, with the annular convolutes easily identified as vertical banding in Figure 4a. The three laser cut notches are clearly identified in the data for the unwrapped hose and are also visible in Figure 4b, although background variations due to braid and lift-off effects somewhat mask the flaws. In obtaining Figure 4c, a twodimensional Fourier transform was applied to Figure $4 \mathrm{~b}$. In the frequency spectrum, the regular spatial pattern of the braided overwrap produced an easily identifiable and separable signature. These components, along with high and low frequency variations, were then removed from the frequency space spectrum. Performing an inverse Fourier Transform on the filtered frequency spectrum produced Figure 4c, which displays the real component of this inverse transform. The automated filtering technique was seen to reduce braid artifacts, improving the signal to noise ratio for the three validation notches.

\section{PROTOTYPE DEVELOPMENT}

Following the success of the initial testing, a scanning fixture was designed to enable eddy current $\mathrm{C}$-scan data to be acquired on installed, pressurized flex hose components. The design, pictured in Figure 5, incorporated a spring loaded probe holder integrated into a manual scanner. The scanner can be coupled directly to the flex hose with a minimal pressure of approximately 1 psi. An optical encoder was used to track probe position along the axis of the hose, with the circumferential position around the hose indexed at a resolution of 2.5 degrees (approximately 0.022 " for 1 " diameter hose). A ball joint coupling between the probe holder and fixed ends allowed scanning of a hose with a radius of curvature of 23 " or greater (hose sections with smaller radius or curvature could be inspected with a restricted scan length along the axis of the hose). This, combined with the small profile of the eddy current scanning system enabled coupling to and scanning of installed flex hose components in confined areas with limited access. 


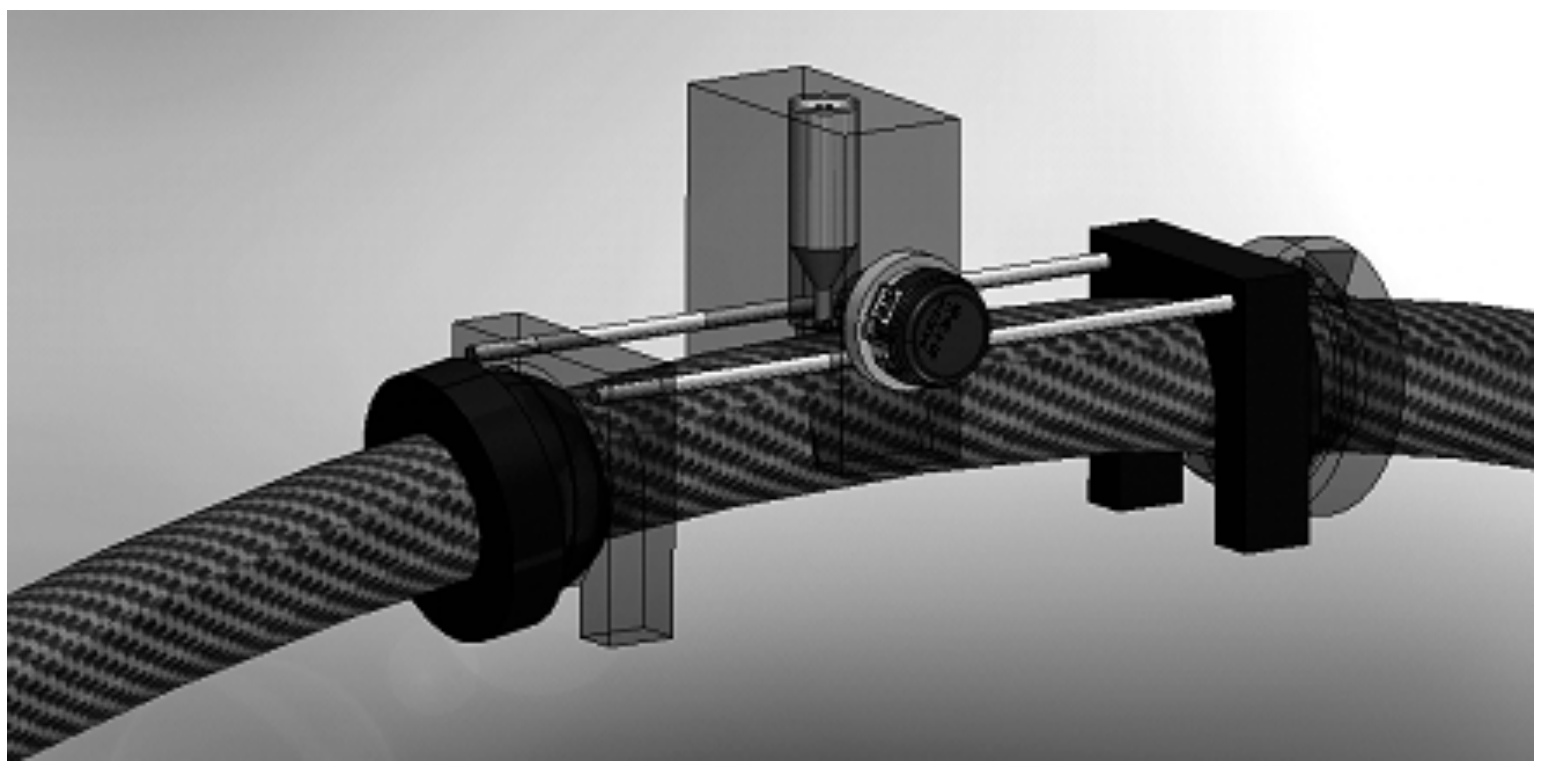

FIGURE 5. Flex hose scanner design for testing of pressurized, installed hoses.

The flex hose scanning system was designed such that the eddy current sensor could be easily changed, without the need to uncouple the scanner from the hose. Along with enabling rapid replacement of a damaged or questionable eddy current coil, this feature allowed for the evaluation of different sensors on an indication of interest. As an example, probes with higher or lower operating frequencies could be applied to add additional information on the depth of the feature causing the indication. In this work the capabilities of a differential sensor with coil spacing equal to the pitch of the corrugation were studied and compared to that of an absolute coil sensor. It was reasoned that a relative measurement between equal areas of adjacent hose corrugations could help to increase the signal to noise by reducing the background levels. Each coil of the differential coil set was designed as described above and pictured in Figure 2. The two coils were then bonded together in a parallel axis configuration. As with the absolute probe, testing of the flex hose was performed with coil axes tangent to the inspection surface and along the convolute direction. Photographs of (a) the differential probe configuration in reference to the corrugation direction and spacing and (b) the flex scanner attached to the validation sample are displayed in Figure 6.

The performance of the flex hose scanning system with the differential eddy current probe is illustrated in Figure 7. The unprocessed vertical channel C-scan of Figure 7a can
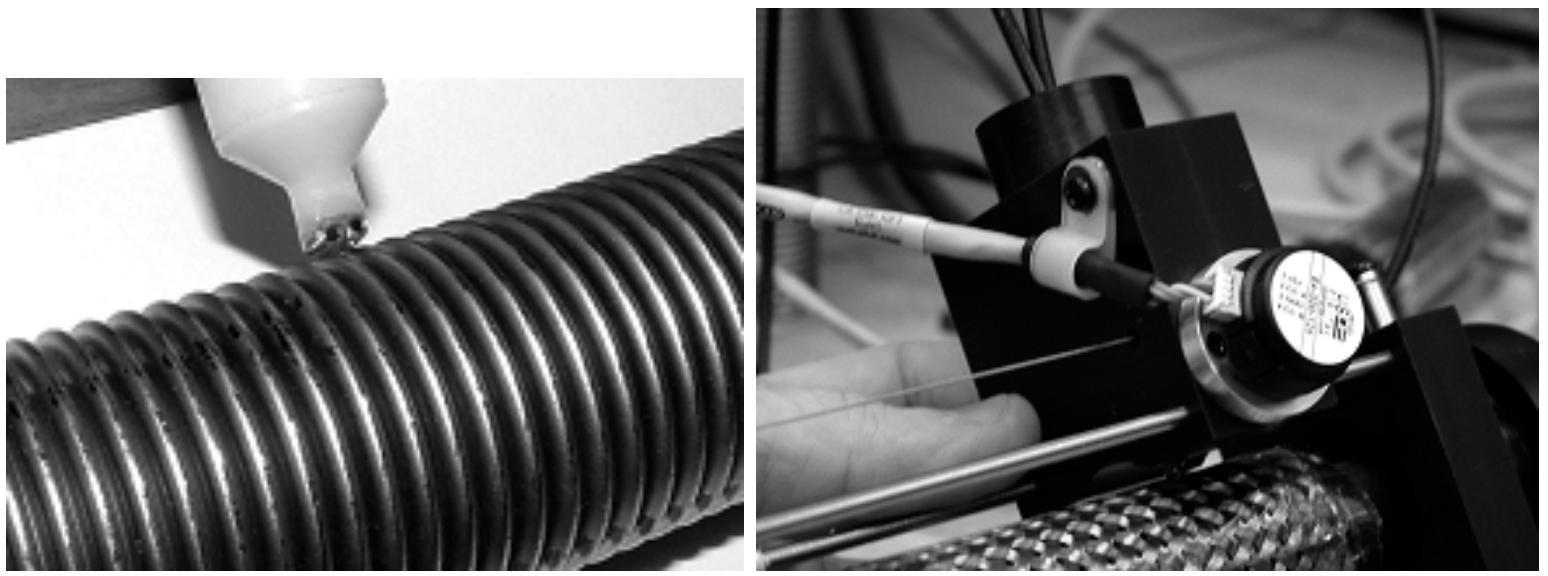

FiguRE 6. Differential eddy current flex hose probe and flex hose scanning system. 
be compared to Figure $4 \mathrm{~b}$, which shows the response from the absolute probe on the same sample. Note that the flex hose orientation is consistent between Figures 4 and 7, but the starting position of the scan is rotated approximately 135 degrees between the two figures. The signal to noise ratio for the system shows great improvement with the differential coil probe. A pronounced maximum-minimum pair is observed at each of the three defects, as expected for differential coil inspection. This characteristic response is highlighted in the extracted lissajous pattern of Figure $7 \mathrm{~b}$. Braid effects are seen to produce a signal response very close to the horizontal axis, with amplitude of approximately $+-2 \mathrm{~V}$. The calibration defect produces a strong vertical response with amplitude more than double that of the braid effect at approximately $+-5 \mathrm{~V}$.

Further improvement to the flaw detection capabilities of the flex hose scanning system was investigated through image processing of the raw $\mathrm{C}$-scan data. The processing routine was designed to use prior knowledge of the braid direction and differential coil orientation and spacing to highlight potential circumferential cracking in the convolute of the flex hose. First, a differential filter along the vector separating the two coils was applied to the raw data. Next, following the image processing procedure presented in reference to Figure 4c, two dimensional Fourier filtering was applied to remove braid artifacts. A morphological opening was then applied using a structural element larger than the coil to coil spacing. As the result of a morphological opening is to highlight objects in the image larger than the structural element, this opening produced a background image of features larger than the coil to coil spacing. The final result, shown in Figure 7c, was obtained by subtracting this background image from that obtained after the two dimensional Fourier filtering. Figure $7 d$ displays a B-scan of the processed amplitude data across a line containing one of the calibration notches. The signal to noise ratio, computed as the quotient of the peak amplitude of the flaw divided by that of the background, is approximately 10 to 1 .

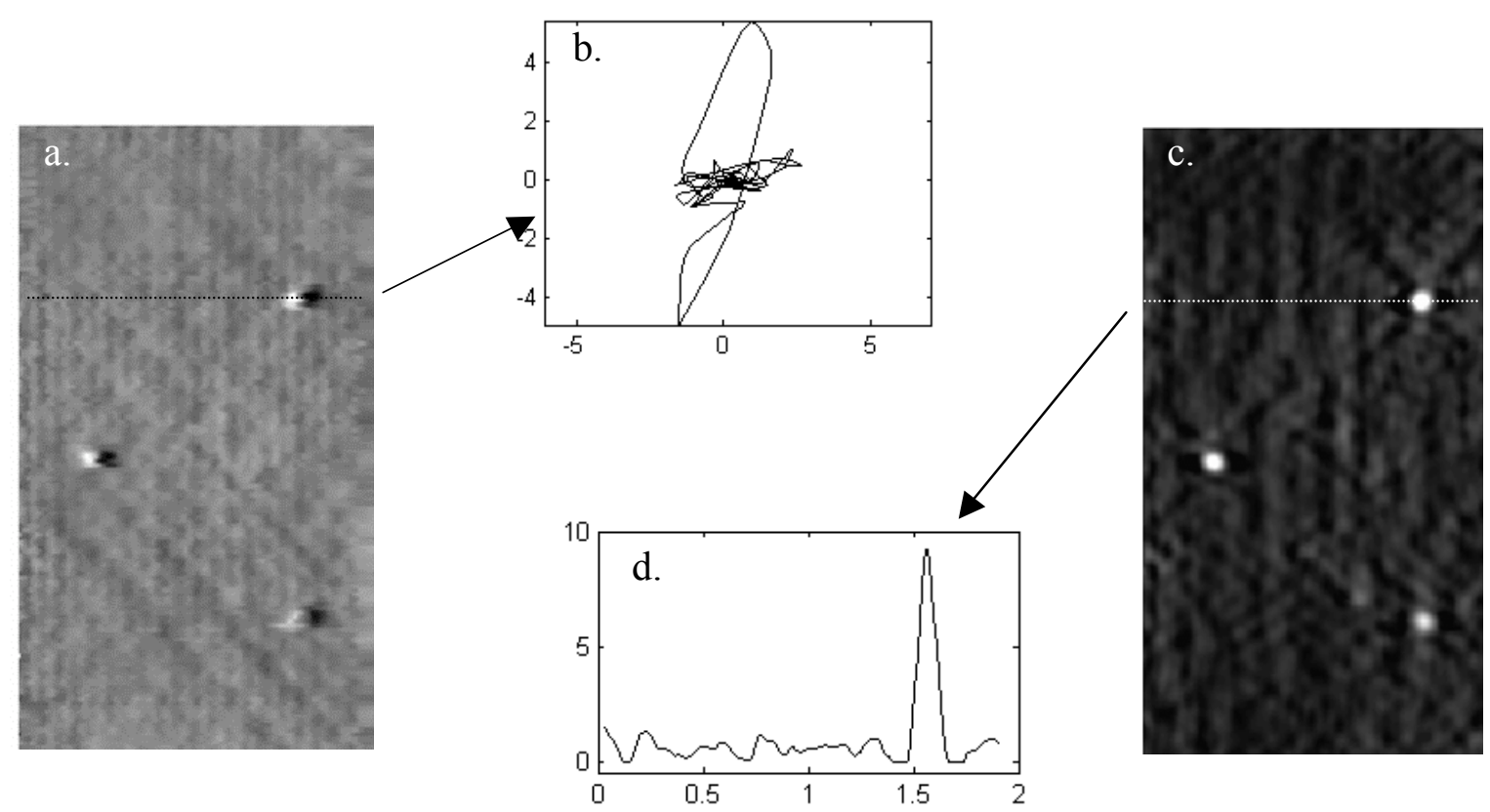

FIGURE 7. (a) Vertical component eddy current C-scan data for validation sample with braided overwrap in place, (b) lissajous pattern extracted from raw data for horizontal line across notch, (c) image processing results of (a), and (d) line scan across calibration artifact in (c). 


\section{SUMMARY}

In this work an eddy current technique for nondestructive evaluation of corrugated metal hose, or flex hose, has been presented. Tangential eddy current sensors, both absolute and differential, were designed and optimized for detection of partially-throughthe-thickness circumferential cracking in the hose convolute through a metallic braid overwrap. The eddy current sensor is incorporated into a flex hose scanning system to enable C-scan data acquisition of in service flex hose components. An image processing routine for the $\mathrm{C}$-scan images based upon prior knowledge of the braid direction and coil parameters was also developed and shown to improve greatly the signal to noise ratio for laser cut notches placed into a validation sample. The capabilities of the prototype system have been examined in reference to a target inspection of the Space Shuttle Radiator Retract Flex Hose. Customization of the technique to meet inspection requirements for other critical flex hose components can be performed based upon the design considerations outlined in this work.

\section{REFERENCES}

1. D. Piotrowski, M. Bode, J. Bohler and D. Moore, "Assessment of Capabilities and Readiness of Conventional and Emerging NDI Methods for Detecting Subsurface Cracks in Lap Joint Structure," $9^{\text {th }}$ Joint FAA/DoD/NASA Aging Aircraft Conference, Atlanta GA, 2006.

2. B. Wincheski, P. Williams, and J. Simpson, "Analysis of Eddy Current Capabilities for the Detection of Outer Diameter Cracking in Small Bore Metallic Structures," in Review of Progress in QNDE, 27A, edited by D. O. Thompson and D. E. Chimenti, AIP Conference Proceedings vol. 975, American Institute of Physics, Melville, NY (2008), pp. 384-391.

3. H. Libby, Introduction to Electromagnetic Nondestructive Test Methods, Robert E. Krieger Publishing Company, Inc., Melbourne, Florida, 1971, pp. 122-129

4. NASA, http://www.nasa.gov/returntoflight/system/system_Orbiter.html, 2006.

5. Chris Bergin, "Engineers evaluating radiator hose issue on the fleet," http://www.nasaspaceflight.com, 2007.

6. Chris Bergin, "Engineers find issue with Atlantis' radiator retract hose," http://www.nasaspaceflight.com, 2008. 